\title{
A Spline Group - Korovkin Approximation Theorem
}

\author{
Malik Saad Al-Muhja ${ }^{1,2}$ \\ ${ }^{1}$ Department of Mathematics, College of Mathematics and Computers Science, Kufa University, Al-Najf Al- \\ Ashraff, Iraq \\ ${ }^{2}$ Department of Mathematics and Computer Application, College of Sciences, University of Al-Muthanna, \\ Samawa 66001, Iraq \\ Correspondence: Malik Saad Al-Muhja, College of Sciences, University of Al-Muthanna, Samawa 66001, Iraq. \\ Tel: 9-647-800-675-951. E-mail: malik@mu.edu.iq
}

Received: February 18, 2015 Accepted: March 10, 2015 Online Published: April 8, 2015

doi:10.5539/jmr.v7n2p110 URL: http://dx.doi.org/10.5539/jmr.v7n2p110

\begin{abstract}
In this paper, using homogeneous groups, we prove a Korovkin type approximation theorem for a spline group by using the notion of a generalization of positive linear operator.
\end{abstract}

Keywords: Piecewise polynomial functions, Spline, Lie group, Group homomorphism, Korovkin type theorem, Positive linear operators

\section{Introduction and Preliminaries}

In this work, we prove a Korovkin approximation theorem by applying the notion of spline with homogeneous groups. Several mathematicians have worked on extending or generalizing the Korovkin's theorems in many ways and to several setting, including function spaces, abstract Banach lattices, Banach algebras and Banach spaces. This theory is very useful in real analysis, functional analysis, harmonic analysis, measure theory, probability theory, summability theory and partial differential equations. But the foremost applications are concerned with constructive approximation theory, which uses it as a valuable tool. Even today, the development of Korovkin type approximation theorem is far from complete.

In this field, Mursaleen work as follows:

Statistical lacunary summability and strongly $\theta_{q}$-convergence $(0<q<\infty)$ and establish some relations between lacunary statistical convergence, statistical lacunary summability and strongly $\theta_{q}$-convergence (Mursaleen \& Alotaibi, 2011, pp. 373-381). And introduce provided a Korovkin type approximation theorem by using the test functions $1, e^{-x}, e^{-2 x}$ here the rate of statistical summability $(C, 1)$ and apply the classical Baskakov operator (Mohiuddine, Alotaibi \& Mursaleen, 2012), also he study the rate of functions of two variables through statistical $A$-summability to Korovkin second theorem via statistical summability $(C, 1)$ (Mursaleen, \& Alotaibi, 2012). Recently, (Ergur, \& Duman, 2013) they have defined generalize and develop the Korovkin type approximation theory by using an appropriate abstract space. This was done, presented a Korovkin type theorem for an interleave between Riesz's representation theory and Lebesgue-Stieltjes integral-i, for Riesz's functional supremum formula via statistical limit (Al-Muhja, 2014). Malliavin calculus, initiated by (Graczyk, 1991, pp. 183-205), was a stable semigroup of measures on homogeneous Lie groups are a natural generalization of the notion of a strictly stable measure on $\mathcal{R}^{n}$.

First of all, we recall some will accept the notes and definitions used in this paper. The concept of a Korovkin approximation for positive linear operator was introduced by (Fast, 1951, pp. 241-244) and further studied many others. Let $K \subseteq \mathbb{N}$, the $A$-density of $K$ denoted by $\delta_{A}(K)$ is defined to be $\delta_{A}(K)=\lim _{j} \sum_{n \in K} a_{j n}$ provided that the limit exists. Using this $A$-density, we say that a sequence $x=\left(x_{n}\right)$ is $A$-statistically convergent to $L$ if and only if

$$
\delta_{A}(K(\epsilon))=0 \text { for every } \epsilon>0 \text {, where } K(\epsilon)=\left\{n \in \mathbb{N}:\left|x_{n}-L\right| \geq \epsilon\right\}
$$

or, equivalently

$$
\lim _{j} \sum_{n:\left|x_{n}-L\right| \geq \epsilon} a_{j n}=0 .
$$

This limit is denoted by $s t_{A}-\lim _{n} x_{n}=L$. 
For a given sequence $\left(x_{n}\right)$, the $A$-transform $x$ denoted by $\left((A x)_{j}\right)$ is given by $(A x)_{j}=\sum_{n=1}^{\infty} a_{j n} x_{n}$ provided the series converges for each $j \in \mathbb{N}$. We say that $A$ is regular if $\lim _{j}(A x)_{j}=L$, whenever $\lim _{n} x_{n}=$ $L$.

Theorem 1.1 (Altomare \& Campiti, 1994) An infinite summability matrix $A=\left(a_{j n}\right)$ is regular if and only if it satisfies all of the following properties

$$
\begin{gathered}
\sup _{j} \sum_{n=1}^{\infty}\left|a_{j n}\right|<\infty, \\
\lim _{j} a_{j n}=0 \text { for each } n \in \mathbb{N}, \\
\lim _{j} \sum_{n=1}^{\infty} a_{j n}=1 .
\end{gathered}
$$

The classical Korovkin approximation theorem is stated as follows (Korovkin, 1960):

Theorem 1.2 Let $\left(T_{n}\right)$ be a sequence of positive linear operators from $C[a, b]$ into $C[a, b]$. Then $\lim _{n}\left\|T_{n}(f, x)-f(x)\right\|_{\infty}=0$, for all $f \in C[a, b]$ if and only if $\lim _{n}\left\|T_{n}\left(f_{i}, x\right)-f_{i}(x)\right\|_{\infty}=0$, for $i=0,1,2$, where $f_{0}(x)=1, f_{1}(x)=x$ and $f_{2}(x)=x^{2}$.

\section{Spline Group and Operator}

The problem of this paper is to study a spline using some properties a Korovkin approximation theorem (Kopotun, 2006, pp. 36-43; Kopotun, 2007, pp. 913-945; Fast, 1951, pp. 241-244), as well as the use of homogeneous groups (Graczyk, 1991, pp. 183-205):

Let $s$ be piecewise polynomial of degree $\leq r$ on $t_{n}$ such that $s \in C^{v-1}[-1,1]$, where $t_{n}=\left(\cos \frac{(n-i) \pi}{n}\right)_{i=0}^{n}$, $n \in \mathbb{N}, 1 \leq v \leq r$ is the Chebyshev partition of $[-1,1]$.

Let $\mathcal{S}_{r}\left(\mathrm{z}_{n}\right)$ be the space of all piecewise polynomial functions ( which we refer to as "splines" ) of degree $r$ ( order $r+1$ ), with the knots $\mathrm{z}_{n}=\left(\mathrm{z}_{i}\right)_{i=0}^{n}$,

$$
-1=\mathrm{z}_{0}<\mathrm{z}_{1}<\cdots<\mathrm{z}_{n-1}<\mathrm{z}_{n}=1 .
$$

In other words, $s \in \mathcal{S}_{r}\left(\mathrm{z}_{n}\right)$ if, on each interval $\left(\mathrm{z}_{i}, \mathrm{z}_{i+1}\right), 0 \leq i \leq n-1, s$ is in $\pi_{r}$, where $\pi_{r}$ denotes the space of algebraic polynomials of degree $\leq r$.

Let $G$ be a family of all functions, and $(G, \circ)$ be a group, such that

$$
G:=\left\{s \in \mathcal{S}_{r}\left(\mathrm{z}_{n}\right): \exists \tau \in \mathcal{S}_{r}\left(\mathrm{z}_{n}\right) \quad \ni s \circ \tau \text { is a continuous function, } \mathrm{z}_{n}=\left(\mathrm{z}_{i}\right)_{i=0}^{n}\right\},
$$

we also denote $(G, \circ)=\left(G_{s}, \circ\right)$. It is well known that $\left(G_{s}, \circ\right)$, is said to be spline group. We define the norm on $\mathcal{S}_{r}\left(\mathrm{z}_{n}\right)$, by formula $\left\|G_{s}\right\|_{S_{r}\left(\mathrm{z}_{n}\right)}:=\sup _{s \in \mathcal{S}_{r}\left(\mathrm{z}_{n}\right)}\left|G_{s}(s)\right|$. Let $\left(G_{s}, \circ,\|\cdot\|\right)$ be a norm group with the norm function $\|\cdot\|: G_{s} \rightarrow[0, \infty)$. Given $\epsilon>0$, does there exist a $\delta(\epsilon)>0$, such that if a mapping

$$
\Upsilon: G_{s} \rightarrow G_{s}
$$

satisfies the inequality

$$
\|\Upsilon(s \circ \tau)-\Upsilon(s) \circ \Upsilon(\tau)\|_{S_{r}\left(\mathrm{z}_{n}\right)}<\delta,
$$

for all $s, \tau \in G_{s}$, then there is a homomorphism

$$
\Upsilon: G_{s} \rightarrow G_{s}
$$

with

$$
\left\|\Upsilon\left(G_{s} ; \cdot\right)-G_{s}(\cdot)\right\|_{S_{r}\left(\mathrm{z}_{n}\right)}<\epsilon, \text { for all } s \in G_{s} .
$$

A spline group $G_{s}$, endowed with the Lie product

$$
\begin{gathered}
G_{s} \times G_{s} \rightarrow G_{s}: m(s, \tau) \mapsto s \circ \tau, \\
G_{s} \rightarrow G_{s}: i(\tau) \mapsto \tau^{-1}
\end{gathered}
$$

on $G_{s}$, is called a spline Lie group (Cho, Saadati, \& Vahidi, 2012; Carter, Segal, \& Macdonald, 1995; Kirillov, 2008).

Hence, we introduce:

Let $G_{s}$ be a spline Lie group. A mapping $\mathcal{H}: G_{s} \rightarrow G_{s}$ is called a spline Lie group homomorphism if $\mathcal{H}$ is 
satisfies (6), and group homomorphism for all $s, \tau \in G_{s}$.

Since $\left(G_{S}, \circ\right)$ is denote a spline group. Hence, in this paper, we define the operator by using (3) with formula:-

Let $G_{s}$ be a spline Lie group. A family $\mho_{\tau}$, where $\tau(\cdot)>0$, of probability measures on $G_{s}$ is called a continuous semigroup of measures if

$$
\mho_{s} * \mho_{\tau}=\mho_{s \circ \tau}
$$

where $s, \tau$ is positive spline,

$$
\mho_{\tau} \Rightarrow I_{e} \quad \text { as } \quad \tau=1,
$$

where $I_{e}$ is identity to spline group and a family of dilations $\left\{\gamma_{\tau}\right\}_{\tau>0}$ is said to be a homogeneous group.

A spline $s$ in $G_{s} /\left\{I_{e}\right\}$ is called homogeneous of degree $\lambda \in \mathcal{R}$ if

$$
s \circ \gamma_{\tau}=\tau^{\lambda} s \text { for } \tau>0 .
$$

A distribution $\sigma$ on $G_{s}$ is homogeneous of degree $\lambda$ if

$$
\left\langle\sigma,\left(\tau^{-\sum_{i=1}^{\eta} d_{i}}\right) s \circ \gamma_{\tau-1}\right\rangle=\tau^{\lambda}\langle\sigma, s\rangle,
$$

for $s \in G_{s}, d_{i} \in \mathcal{R}, \eta \in \mathbb{N}$ and $\tau>0$.

A linear differential operator $\Upsilon$ on $G_{s}$ is homogeneous of degree $\lambda$ if

$$
\Upsilon\left(s \circ \gamma_{\tau}\right)=\tau^{\lambda}(\Upsilon s) \circ \gamma_{\tau},
$$

for any $s \in G_{s}$ and $\tau>0$.

We will a generalization of positive linear operator $\Upsilon_{j}$ by using our Lemma as follow:

Lemma 2.1 (A-statistically homogeneous group) Let $A=\left(a_{j n}\right)$ be nonnegative regular summability matrix. For all $s \in G_{s} /\left\{I_{e}\right\}$, satisfied (3), and $\Upsilon_{j}$ a sequence of positive linear operators, we have $\Upsilon_{j}: G_{s} /\left\{I_{e}\right\} \rightarrow G_{s} /\left\{I_{e}\right\}$, homogeneous group.

Proof. For $s \in G_{s} /\left\{I_{e}\right\}$, by using (4), (9) and (11), it is clear that,

$$
\begin{aligned}
& s t-\lim _{j} \sum_{n=1}^{\infty} a_{j n}\left\|\Upsilon_{j}\left(s \circ \gamma_{\tau}\right)-\Upsilon_{j}(s) \circ \Upsilon_{j}\left(\gamma_{\tau}\right)\right\|_{\mathcal{S}_{r}\left(\mathrm{z}_{n}\right)} \\
& =s t-\lim _{j} \sum_{n=1}^{\infty} a_{j n}\left\|\tau^{\lambda}\left(\Upsilon_{j} s\right) \circ \gamma_{\tau}-\Upsilon_{j}(s) \circ \gamma_{\tau}\right\|_{\mathcal{S}_{r}\left(\mathrm{z}_{n}\right)} \\
& =s t-\lim _{j} \sum_{n=1}^{\infty} a_{j n}\left\|\tau^{\lambda}\left(\Upsilon_{j} s\right) \circ \gamma_{\tau}-\frac{\tau^{\lambda}}{\tau^{\lambda}}\left(\Upsilon_{j} s\right) \circ \gamma_{\tau}\right\|_{\mathcal{S}_{r}\left(\mathrm{z}_{n}\right)} \\
& =s t-\lim _{j} \sum_{n=1}^{\infty} a_{j n}\left\|\left(1-\frac{1}{\tau^{\lambda}}\right) \tau^{\lambda}\left(\Upsilon_{j} s\right) \circ \gamma_{\tau}\right\|_{\mathcal{S}_{r}\left(\mathrm{z}_{n}\right)} \\
& =s t-\lim _{j} \sum_{n=1}^{\infty} a_{j n}\left\|\left(1-\frac{1}{\tau^{\lambda}}\right) \tau^{\lambda}\left(\Upsilon_{j} s\right) \circ \gamma_{\tau}\right\|_{\mathcal{S}_{r}\left(\mathrm{z}_{n}\right)} \\
& =0
\end{aligned}
$$

hence from (1), we have

$$
\delta_{A}(K)=0 .
$$

Therefore the Lemma is proved, for $s \in G_{s} /\left\{I_{e}\right\}$.

In other words, if a sequence $\Upsilon_{j}$ is a homomorphism from $G_{s}$ into $G_{s}$, then $\Upsilon_{j}: G_{s} /\left\{I_{e}\right\} \rightarrow G_{s} /\left\{I_{e}\right\}$, is 
homogeneous group and A-statistically convergent.

\section{Group and Korovkin Theorem}

Now, we present the following results:

Theorem 3.1 Let $A=\left(a_{j n}\right)$ be nonnegative regular summability matrix, and let $\Upsilon_{j}$ be a sequence of positive linear operators from $\mathcal{S}_{r}\left(z_{n}\right)$ into $\mathcal{S}_{r}\left(z_{n}\right)$. Then for all $s \in \mathcal{S}_{r}\left(z_{n}\right)$, we have

$$
s t-\lim _{j} \sum_{n=1}^{\infty} a_{j n}\left\|Y_{j}\left(G_{s} ; \cdot\right)-G_{s}\right\|_{\delta_{r}\left(\mathrm{z}_{n}\right)}=0
$$

if and only if

$$
\begin{array}{ll}
s \circ s_{\circ}=1, & \ni s_{\circ} \in G_{s \circ}, \\
G_{s}(s \circ \tau)=\tau^{\lambda} s, & \ni \tau \in G_{s 1}, \\
G_{s}(s \circ v)=s \circ \gamma_{\tau-1}, & \ni v \in G_{s 2} ;
\end{array}
$$

such that

$$
s t-\lim _{j} \sum_{n=1}^{\infty} a_{j n}\left\|Y_{j}\left(G_{s i} ; .\right)-G_{s i}\right\|_{S_{r}\left(\mathrm{z}_{n}\right)}=0 \quad ; \quad i=0,1,2
$$

and $G_{s i}$ is a subgroup from $G_{s} ; i=0,1,2$.

Proof. Since each of $1, \tau^{\lambda} s, s \circ \gamma_{\tau-1}$ belongs to $\mathcal{S}_{r}\left(\mathrm{z}_{n}\right)$, condition (15), follow immediately from (13). Now, assume that (15) holds. For the splines $\tau, v$ given in (14), we get

$$
\left|G_{s}(t)-G_{s}(s)\right|<\epsilon+\vartheta\left(\mho_{\tau}\right)
$$

where

$$
\begin{gathered}
\vartheta\left(\mho_{\tau}\right)=\left(\tau^{\lambda} t\right)-\left(t \circ \gamma_{\tau-1}\right) \\
=G_{S}(t \circ \tau)-G_{s}(t \circ v) .
\end{gathered}
$$

This is,

$$
-\epsilon-\vartheta\left(\mho_{\tau}\right)<G_{s}(t)-G_{s}(s)<\epsilon+\vartheta\left(\mho_{\tau}\right) .
$$

Now, applying the operators $\Upsilon_{j}\left(s \circ S_{\circ} ; s\right)$ for every $j \in \mathbb{N}$, to this inequality,

$$
\begin{aligned}
-\epsilon \Upsilon_{j}\left(s \circ S_{\circ} ; s\right)- & \vartheta\left(\mho_{\tau}\right) \Upsilon_{j}\left(s \circ S_{\circ} ; s\right)<\Upsilon_{j}\left(G_{s}(t) ; s\right)-G_{s}(s) \Upsilon_{j}\left(s \circ S_{\circ} ; s\right) \\
& <\epsilon \Upsilon_{j}\left(s \circ S_{\circ} ; s\right)+\vartheta\left(\mho_{\tau}\right) \Upsilon_{j}\left(s \circ S_{\circ} ; s\right)
\end{aligned}
$$

also,

$$
\begin{gathered}
\Upsilon_{j}\left(G_{s}(t) ; s\right)-G_{s}(s)=\Upsilon_{j}\left(G_{s}(t) ; s\right)-G_{s}(s) \Upsilon_{j}\left(s \circ s_{o} ; s\right)+G_{s}(s) \Upsilon_{j}\left(s \circ s_{\circ} ; s\right)-G_{s}(s) \\
=\Upsilon_{j}\left(G_{s}(t) ; s\right)-G_{s}(s) \Upsilon_{j}\left(s \circ s_{\circ} ; s\right)+G_{s}(s)\left[\Upsilon_{j}(1 ; s)-1\right] .
\end{gathered}
$$

If follows from (17) and (18), that

Now, from (16)

$$
\begin{aligned}
\Upsilon_{j}\left(G_{s}(t) ; s\right) & -G_{s}(s)<\epsilon \Upsilon_{j}\left(s \circ S_{\circ} ; s\right)+\vartheta\left(\mho_{\tau}\right) \Upsilon_{j}\left(s \circ S_{\circ} ; s\right)+G_{s}(s)\left[\Upsilon_{j}(1 ; s)-1\right] \\
& <\epsilon \Upsilon_{j}\left(s \circ S_{\circ} ; s\right)+\Upsilon_{j}\left(\vartheta\left(\mho_{\tau}\right) ; s\right)+G_{s}(s)\left[\Upsilon_{j}(1 ; s)-1\right] .
\end{aligned}
$$

$$
\begin{gathered}
\Upsilon_{j}\left(\vartheta\left(\mho_{\tau}\right) ; s\right)=M\left(\Upsilon_{j}\left(G_{s}(t \circ \tau)-G_{s}(t \circ v) ; s\right)\right) \\
=M\left(\Upsilon_{j}\left(G_{s}(t \circ \tau) ; s\right)-\Upsilon_{j}\left(G_{s}(t \circ v) ; s\right)\right),
\end{gathered}
$$

where $M=\epsilon+1$.

Using (19), we obtain

$$
\begin{gathered}
\Upsilon_{j}\left(G_{s}(t) ; s\right)-G_{s}(s)<\epsilon \Upsilon_{j}\left(s \circ s_{\circ} ; s\right)+M\left(\Upsilon_{j}\left(G_{s}(t \circ \tau) ; s\right)-G_{s}(s)+G_{s}(s)-\Upsilon_{j}\left(G_{s}(t \circ v) ; s\right)\right)+ \\
G_{s}(s)\left[\Upsilon_{j}(1 ; s)-1\right] \\
<\epsilon\left(\Upsilon_{j}\left(s \circ s_{\circ} ; s\right)-\left(s \circ s_{\circ}\right)+\left(s \circ s_{\circ}\right)\right)+M\left(\Upsilon_{j}\left(G_{s}(t \circ \tau) ; s\right)-G_{s}(s)+G_{s}(s)-\right.
\end{gathered}
$$




$$
\left.\Upsilon_{j}\left(G_{s}(t \circ v) ; s\right)\right)+G_{s}(s)\left[\Upsilon_{j}(1 ; s)-1\right]
$$

Therefore,

$$
\begin{gathered}
\left|\Upsilon_{j}\left(G_{S}(t) ; s\right)-G_{s}(s)\right|<\epsilon\left|\Upsilon_{j}\left(s \circ s_{o} ; s\right)-\left(s \circ s_{\circ}\right)\right|+\epsilon+M\left(\left|\Upsilon_{j}\left(G_{s}(t \circ \tau) ; s\right)-G_{s}(s)\right|+\right. \\
\left.\left|\Upsilon_{j}\left(G_{s}(t \circ v) ; s\right)-G_{s}(s)\right|\right)+\left|G_{s}(s)\right|\left|\Upsilon_{j}(1 ; s)-1\right|,
\end{gathered}
$$

since $\left|G_{s}(s)\right| \leq 1$ for all $s \in G_{s}$ and (14). Now, taking supremum over $s$, we get

$$
\begin{aligned}
& \left\|Y_{j}\left(G_{s}(t) ; s\right)-G_{s}(s)\right\|_{\mathcal{S}_{r}\left(\mathrm{z}_{n}\right)} \leq \epsilon+\epsilon\left\|\Upsilon_{j}(1 ; s)-1\right\|_{\mathcal{S}_{r}\left(\mathrm{z}_{n}\right)}+M\left\|\Upsilon_{j}\left(G_{s}(t \circ \tau) ; s\right)-G_{s}(s)\right\|_{\mathcal{S}_{r}\left(\mathrm{z}_{n}\right)}+ \\
& M\left\|\Upsilon_{j}\left(G_{s}(t \circ v) ; s\right)-G_{s}(s)\right\|_{\delta_{r}\left(\mathrm{z}_{n}\right)}+\left\|\Upsilon_{j}(1 ; s)-1\right\|_{\mathcal{S}_{r}\left(\mathrm{z}_{n}\right)} \\
& \leq \epsilon+(\epsilon+1)\left\|Y_{j}(1 ; s)-1\right\|_{\delta_{r}\left(\mathrm{z}_{n}\right)}+M\left\|\Upsilon_{j}\left(G_{s}(t \circ \tau) ; s\right)-G_{s}(s)\right\|_{\mathcal{S}_{r}\left(\mathrm{z}_{n}\right)}+ \\
& M\left\|\Upsilon_{j}\left(G_{s}(t \circ v) ; s\right)-G_{s}(s)\right\|_{S_{r}\left(\mathrm{z}_{n}\right)} \\
& <\epsilon+M\left[\left\|\Upsilon_{j}(1 ; s)-1\right\|_{\delta_{r}\left(\mathrm{z}_{n}\right)}+\left\|\Upsilon_{j}\left(G_{s}(t \circ \tau) ; s\right)-G_{s}(s)\right\|_{\mathcal{S}_{r}\left(\mathrm{z}_{n}\right)}+\right. \\
& \left.\left\|Y_{j}\left(G_{s}(t \circ v) ; s\right)-G_{s}(s)\right\|_{\mathcal{S}_{r}\left(\mathrm{z}_{n}\right)}\right] .
\end{aligned}
$$

For a given $\xi>0$, choose $\dot{\epsilon}>0$ such that $\dot{\epsilon}<\xi$. Define the following sets,

$$
\begin{gathered}
D=\left\{j:\left\|\Upsilon_{j}\left(G_{s}(t) ; s\right)-G_{s}(s)\right\|_{\mathcal{S}_{r}\left(\mathrm{z}_{n}\right)} \geq \xi\right\}, \\
D_{\circ}=\left\{j:\left\|\Upsilon_{j}(1 ; s)-1\right\|_{\mathcal{S}_{r}\left(\mathrm{z}_{n}\right)} \geq \frac{\xi-\epsilon}{3}\right\}, \\
D_{1}=\left\{j:\left\|\Upsilon_{j}\left(G_{s}(t \circ \tau) ; s\right)-\tau^{\lambda} s\right\|_{\mathcal{S}_{r}\left(\mathrm{z}_{n}\right)} \geq \frac{\xi-\epsilon}{3}\right\}, \\
D_{2}=\left\{j:\left\|\Upsilon_{j}\left(G_{s}(t \circ v) ; s\right)-s \circ \gamma_{\tau-1}\right\|_{\mathcal{S}_{r}\left(\mathrm{z}_{n}\right)} \geq \frac{\xi-\dot{\epsilon}}{3}\right\} .
\end{gathered}
$$

Then $D \subset D_{\circ} \cup D_{1} \cup D_{2}$ and $\delta_{A}(D) \leq \delta_{A}\left(D_{\circ}\right)+\delta_{A}\left(D_{1}\right)+\delta_{A}\left(D_{2}\right)$. Therefore, using conditions (14) and (15), we have

$$
s t-\lim _{j} \sum_{n=1}^{\infty} a_{j n}\left\|\Upsilon_{j}\left(G_{s} ; .\right)-G_{s}\right\|_{\delta_{r}\left(\mathrm{z}_{n}\right)}=0 .
$$

This completes the proof of the theorem.

Theorem 3.2 If $\left(z_{n i}\right)_{i=0}^{\infty}$ is defined by (2), then there exists a set $\mathfrak{J}=\left\{z_{n 0}<z_{n 1}<\cdots<z_{n m}<\cdots\right\} \subseteq \mathbb{N}$, such that $\delta_{\theta}(\mathfrak{J})=1$ and $\Upsilon_{j}$ homogeneous group if and only if a sequence $\Upsilon=\left(\Upsilon_{j}\right)$ is lacunary statistically convergence to $L$.

\section{Auxiliary Results and Proof Theorem 3.2}

Recently the concept of lacunary statistically convergence:

Let $K \subseteq \mathbb{N}$. Then

$$
\delta_{\theta}(K)=\lim _{\dot{r}} \frac{1}{h_{\dot{r}}}\left|\left\{k_{\dot{r}-1}<i<k_{\dot{r}}: i \in K\right\}\right|
$$

is said to be $\theta$-density of $K$.

A sequence $x=\left(x_{k}\right)$ is said to be lacunary statistically convergent (Fridy, \& Orhan, 1993, pp. 43-51) to $L$, if for every $\epsilon>0$, the set $K_{\epsilon}:=\left\{k \in \mathbb{N}:\left|x_{k}-L\right| \geq \epsilon\right\}$ has $\theta$-density zero, i.e. $\delta_{\theta}\left(K_{\epsilon}\right)=0$. In this case we write 
$S_{\theta}-\lim x=L$. That is,

$$
\lim _{\dot{r}}\left|\left\{k_{\dot{r}-1}<i<k_{\dot{r}}:\left|x_{k}-L\right| \geq \epsilon\right\}\right|=0 .
$$

In this case, we write $S_{\theta}-\lim _{i} x_{i}=L$, and we denote the set of all lacunary statistically convergent sequence by $S_{\theta}$.

The following theorems were proved by (Mursaleen \& Alotaibi, 2011, pp. 373-381):

Theorem 4.1 If a sequence $x=\left(x_{k}\right)$ is bounded and lacunary statistically convergent to $L$ then it is statistically lacunary summable to $L$.

Theorem 4.2 (a) If $0<q<\infty$ and a sequence $x=\left(x_{k}\right)$ is strongly $\theta_{q}$-convergent to the limit $L$, then it is lacunary statistically convergent to $L$.

(b) If $x=\left(x_{k}\right)$ is bounded and lacunary statistically convergent to $L$, then $x_{k} \rightarrow L\left|C_{\theta}\right|_{q}$.

Theorem 4.3 A sequence $x=\left(x_{k}\right)$ is statistically lacunary summable to $L$ if and only if there exists a set $K=\left\{r_{1}<r_{2}<\cdots<r_{n}<\cdots\right\} \subseteq \mathbb{N}$, such that $\delta(K)=1$ and $\theta-\lim x_{r_{n}}=L$.

Theorem 4.4 A sequence $x=\left(x_{k}\right)$ is lacunary statistically convergent to $L$ if and only if there exists a set $K=\left\{k_{1}<k_{2}<\cdots<k_{n}<\cdots\right\} \subseteq \mathbb{N}$, such that $\delta_{\theta}(K)=1$ and $\lim x_{k_{n}}=L$.

Now, we prove our theorem:

Proof theorem 3.2 Assume that $t_{n}$ is the Chebyshev partition of $[0, \infty]$. Suppose that $\mathfrak{J} \subseteq \mathbb{N}$, such that $\delta_{\theta}(\mathfrak{J})=1$ and $\Upsilon_{j}$ homogeneous group holds. Then, from Lemma 2.1 and (10) there is a positive integer $\eta$, we have

$$
\begin{gathered}
0 \leftarrow \frac{1}{h_{\dot{\eta}}} \sum_{\dot{\eta}=-\sum_{i=1}^{\eta} d_{i}}\left|\left\langle\sigma, \tau^{\dot{\eta}} s \circ \gamma_{\tau-1}\right\rangle\right|^{2}>\frac{1}{h_{\dot{\eta}}} \sum_{\dot{\eta}=-\sum_{i=1}^{\eta} d_{i}}|\sigma-L| .\left|\left(\tau^{\dot{\eta}}\left(\Upsilon_{j} s\right) \circ \gamma_{\tau-1}\right)-L\right| \\
>\frac{\epsilon}{h_{\dot{\eta}}} \sum_{\dot{\eta}=-\sum_{i=1}^{\eta} d_{i}}\left|\left(\tau^{\dot{\eta}}\left(\Upsilon_{j} s\right) \circ \gamma_{\tau-1}\right)-L\right| .
\end{gathered}
$$

Put $\mathfrak{I}_{\epsilon}:=\left\{\eta \in \mathbb{N}:\left|\bar{\Upsilon}_{j}-L\right| \geq \epsilon\right\}$, where $\bar{\Upsilon}_{j}=\left(\tau^{\dot{\eta}}\left(\Upsilon_{j} s\right) \circ \gamma_{\tau-1}\right)$ and $\mathfrak{\mathfrak { J }}=\left\{\mathrm{z}_{\eta+1}, \mathrm{z}_{\eta+2}, \ldots\right\}$. Then $\delta_{\theta}(\mathfrak{\mathfrak { J }})=1$ and $\mathfrak{I}_{\epsilon} \subseteq \mathbb{N}-\mathfrak{\mathfrak { I }}$, therefore $\lim _{\eta} \frac{1}{h_{\dot{\eta}}}\left|\mathfrak{I}_{\epsilon}\right|=0$, from (21), which implies that $\delta_{\theta}\left(\mathfrak{I}_{\epsilon}\right)=0$.

Hence $\Upsilon=\left(\Upsilon_{j}\right)$ is lacunary statistically convergence to $L$.

Conversely, let $\Upsilon=\left(\Upsilon_{j}\right)$ be lacunary statistically convergence to $L$. Since $\left(z_{n i}\right)_{i=0}^{\infty}$ is defined by (2), from theorem 4.4, there exists a set $\mathfrak{I}=\left\{z_{n 0}<z_{n 1}<\cdots<z_{n m}<\cdots\right\} \subseteq \mathbb{N}$, such that $\delta_{\theta}(\mathfrak{J})=1$. Now, from (12), we have $\Upsilon_{j}$ is homogeneous group. This completes the proof of the theorem.

Now, we can write more of corollary with using theorems 4.1-4, and theorem 3.2.

Corollary 4.5 A sequence $\Upsilon=\left(\Upsilon_{j}\right)$ is lacunary statistically convergent to $L$, then there exists a set $\mathfrak{I}=$ $\left\{z_{n 0}<z_{n 1}<\cdots<z_{n m}<\cdots\right\} \subseteq \mathbb{N}$, such that $\delta_{\theta}(\mathfrak{J})=1$ and satisfies (1).

Proof. By using theorem 3.2 and Lemma 2.1, it is easily prove that.

Corollary 4.6 If $0<q<\infty$ and a sequence $\Upsilon=\left(\Upsilon_{j}\right)$ is strongly $\theta_{q}$-convergent to the limit $L$, if and only if there exists a set $\mathfrak{I}=\left\{z_{n 0}<z_{n 1}<\cdots<z_{n m}<\cdots\right\} \subseteq \mathbb{N}$, such that $\delta_{\theta}(\mathfrak{I})=1$ and $\Upsilon_{j}$ homogeneous group.

Proof. From Theorem 4.2 (a) and theorem 3.2, it is easily prove that.

\section{Acknowledgements}

The author is grateful for hospitality at the University of Kufa. He thanks his fellows for the fruitful discussions while preparing this paper.

\section{References}

Al-Muhja, M. S. (2014). A Korovkin type approximation theorem and Its applications. Abstract and Applied Analysis, Article ID 859696, 6 pages. http://dx.doi.org/10.1155/2014/859696

Altomare, F., \& Campiti, M. (1994). Korovkin-type approximation theory and Its applications. Walter de Gruyter 
\& Co., D-10785 Berlin. http://dx.doi.org/10.1515/9783110884586

Carter, R., Segal, G., \& Macdonald, I. (1995). Lectures on Lie groups and Lie algebras. vol. 32 of London Mathematical Society Student Texts, Cambridge University Press, Cambridge. http://dx.doi.org/10.1017/CBO9781139172882

Cho, Y. J., Saadati, R., \& Vahidi, J. (2012). Approximation of homomorphisms and derivations on nonArchimedean Lie $C^{*}$-algebras via fixed point method. Discrete Dynamics in Nature and Society, vol. 2012, Article ID 373904, 9 pages. http://dx.doi.org/10.1155/2012/373904

Ergur, A. A., \& Duman, O. (2013). Generalization of statistical Korovkin theorems. Journal of Applied Mathematics, Article ID 187530, 5 pages. http://dx.doi.org/10.1155/2013/187530

Fast, H. (1951). Sur la convergence statistique. Colloq Math. 2, pp. 241-244. http://dx.doi.org/10.1155/2013/187530

Fridy, J. A., \& Orhan, C. (1993). Lacunary statistical convergence. Pacific Journal of Mathematics, 160, 43-51. http://dx.doi.org/10.2140/pjm.1993.160.43

Graczyk, P. (1991). Malliavin calculus for stable processes on homogeneous groups. Studia Mathematica, 100(3), 183-205.

Kirillov, A. Jr. (2008). An introduction to Lie groups and Lie algebras. vol. 113 of Cambridge Studies in Advanced Mathematics, Cambridge University Press, Cambridge. http://dx.doi.org/10.1017/CBO9780511755156

Kopotun, K. A. (2006). On equivalence of moduli of smoothness of splines in Lp, $0<\mathrm{p}<1$. Journal of Approximation Theory, 143, 36-43. http://dx.doi.org/10.1016/j.jat.2006.03.001

Kopotun, K. A. (2007). Univariate splines: Equivalence of moduli of smoothness and applications. Mathematics of Computation, 76(258), 931-945. http://dx.doi.org/10.1090/S0025-5718-06-01920-X

Korovkin, P. P. (1960). Linear operators and approximation theory. Gordon and Breach, Delhi, India.

Mohiuddine, S. A., Alotaibi, A., \& Mursaleen, M. (2012). Statistical summability ( C,1 ) and a Korovkin type approximation theorem. Journal of Inequalities and Applications, 172. http://dx.doi.org/10.1186/1029-242X-2012-172

Mursaleen, M., \& Alotaibi, A. (2012). Korovkin type approximation theorem for functions of two variables through statistical A-summability. Advances in Difference Equations, 65. http://dx.doi.org/10.1186/1687-1847-2012-65

Mursaleen , M., \& Alotaibi, A. (2011). Statistical lacunary summability and a Korovkin type approximation theorem. Annali dell' Universita'di Ferrara, 57(2), 373-381. http://dx.doi.org/10.1007/s11565-011-0122-8

\section{Copyrights}

Copyright for this article is retained by the author(s), with first publication rights granted to the journal.

This is an open-access article distributed under the terms and conditions of the Creative Commons Attribution license (http://creativecommons.org/licenses/by/3.0/). 